\title{
EXCITON DYNAMICS IN ZnTe BASED SEMIMAGNETIC SEMICONDUCTORS AND CdMnTe QUANTUM WELLS
}

\author{
Nguyen The Khoi, J.A. GaJ \\ Institute of Experimental Physics, Warsaw University \\ Hoża 69, 00-681 Warszawa, Poland \\ G. Karczewski, T. Wojtowicz, J. Kossut \\ Institute of Physics, Polish Academy of Sciences \\ Al. Lotników 32/46, 02-668 Warszawa, Poland \\ AND M. DEMIANIUK \\ Institute of Technical Physics, Military Academy of Technology \\ Kaliskiego 2, 00-908 Warszawa, Poland
}

\begin{abstract}
Inelastic scattering of excitons on LO phonons in ZnTe based bulk semimagnetic semiconductors with low concentration of paramagnetic ions $\left(\mathrm{Mn}^{2+}, \mathrm{Fe}^{2+}\right.$ and $\left.\mathrm{Cr}^{2+}\right)$ was studied. A polarization degree of the LO phonon Raman lines was measured as a function of the exciton splitting in a magnetic field, and allowed us to determine the polarization lifetime (polariton flight time). A similar method was used to investigate multiple $\mathrm{Mn}^{2+}$ spin-flip scattering in CdMnTe quantum well. The cascade model seems to be not adequate to interpret the dependence of the polarization degree on the order of scattering.
\end{abstract}

PACS numbers: 75.50.Pp, 78.66.Hf, 73.20.Dx

\section{Introduction}

The resonant Faraday rotation of excitonic polariton, first observed in CdSe by Nawrocki et al. [1], provides a powerful tool to study scattering processes of polaritons. The polarization lifetime can be measured by using the exciton splitting as an internal clock. When applying the method to semimagnetic semiconductors (SMSCs), we can take advantage from the giant Zeeman splitting arising from the exchange interaction between localized magnetic ions and band carriers. This means that in these materials the internal clock runs very fast and it allows us to measure short times [2]. This effect has been used to study crystals of CdFeSe and $\mathrm{ZnFeSe}[3-5]$, in which cascades due to inelastic scattering on $\mathrm{Fe}^{2+}$ ions, on $\mathrm{LO}$ phonons, and combined scattering on $\mathrm{LO}$ phonons $-\mathrm{Fe}^{2+}$ ions were observed. 


\section{Experiment and results}

\subsection{ZnTe based bulk SMSC}

We report the results obtained in applying the method to the family of ZnTe based SMSCs containing low concentration $(x \leq 1 \%)$ of paramagnetic ions $\left(\mathrm{Mn}^{2+}, \mathrm{Fe}^{2+}\right.$ and $\left.\mathrm{Cr}^{2+}\right)$. We chose a crystal of $\mathrm{ZnMnTe}$ with $x=1 \%$. For the remainders we used the ones with largest concentrations of magnetic ions that were available (about $0.1 \%$ of $\mathrm{Cr}$ and $0.2 \%$ of $\mathrm{Fe}$ ). The exciton energy measured in reflectivity is $19203 \mathrm{~cm}^{-1}$ for $\mathrm{ZnTe}, 19205 \mathrm{~cm}^{-1}$ for $\mathrm{ZnCrTe}, 19208 \mathrm{~cm}^{-1}$ for $\mathrm{ZnMnTe}$ and $19209 \mathrm{~cm}^{-1}$ for ZnFeTe. Raman scattering measurements were performed in backscattering geometry using linearly polarized beam of an Ar laser. The beam power was about $30 \mathrm{~mW}$. The samples were cleaved from bulk crystals. All measurements were done at $2 \mathrm{~K}$ with the samples immersed in pumped liquid helium. The measuring setup was the same as described in Ref. [4]. The complex polarization degree $P$ was determined as a function of exciton splitting.

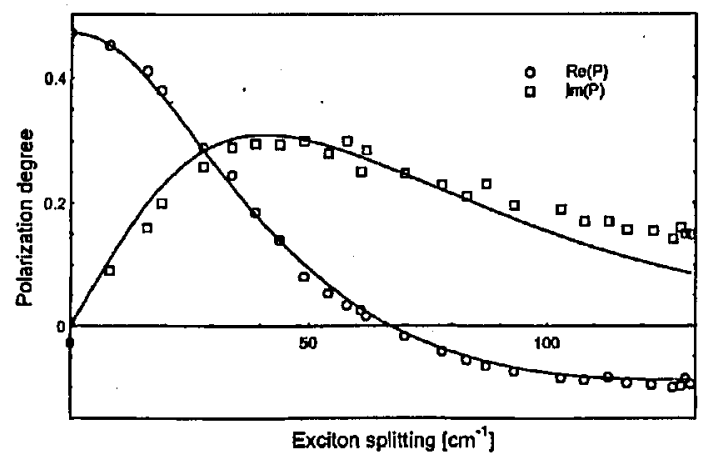

Fig. 1. Real and imaginary parts of the polarization degree of the $1 \mathrm{LO}$ peak in $\mathrm{ZnMnTe}$ vs. exciton Zeeman splitting. The solid curves are the best fit.

By exciting the crystals with the laser line $19436 \mathrm{~cm}^{-1}$ we saw a series of Raman peaks separated by the LO phonon energy $\left(\omega_{\mathrm{LO}}=210 \mathrm{~cm}^{-1}\right.$ in $\left.\mathrm{ZnTe}\right)$. This type of spectra is often explained as resulting from inelastic scattering of the photo-created excitons on LO phonons via a cascade process. For $\mathrm{ZnMnTe}$, the dependence of the real and imaginary parts of $P$ for the 1 LO phonon peak (hereafter called the 1 LO peak) on exciton Zeeman splitting is shown in Fig. 1. In order to determine the characteristic time we used the model proposed by Ivchenko et al. [6] based on a microscopic theory for light scattering which takes into account multiple scattering of excitons. From the best fit of the polarization curves to the model, we obtained the polarization lifetime $T=0.1 \mathrm{ps}$ and the momentum relaxation time $\tau_{\mathrm{p}}=0.02 \mathrm{ps}$. By analogy to previous cases, we interpret the polarization lifetime as the time of flight of excitonic polariton $[1,5]$.

In $\mathrm{ZnFeTe}$ and $\mathrm{ZnCrTe}$ crystals under excitation of the same laser line, we obtained similar polarization dependence of the 1 LO peak. However, because of the small concentrations of magnetic ions, the Zeeman splitting is weaker than 
in $\mathrm{ZnMnTe}$, and we could measure only a fragment of the curves (up to splitting energy values $60 \mathrm{~cm}^{-1}$ in $\mathrm{ZnFeTe}$ and $22 \mathrm{~cm}^{-1}$ in $\mathrm{ZnCrTe}$ ). Nevertheless we could fit the curves by the same way as for $\mathrm{ZnMnTe}$, and obtained $T=0.06 \mathrm{ps}$ for $\mathrm{ZnFeTe}$ and $T=0.07 \mathrm{ps}$ for $\mathrm{ZnCrTe}$. The different values of polarization lifetime may arise from the fact that in each material the depolarization time is different and depends on the nature of the magnetic ions. It is worth noting that in $\mathrm{ZnCrTe}$, when measuring $\operatorname{Im}(P)$, we did observe the opposite sign of the rotation in comparison with that of $\mathrm{ZnMnTe}$ and $\mathrm{ZnFeTe}$. This agrees with the fact that in $\mathrm{Cr}$ based SMSCs, the $p-d$ exchange interaction is ferromagnetic, while in the other ones it is antiferromagnetic [8].

For the $2 \mathrm{LO}$ peak in ZnMnTe we observed certain peculiarities. First, the value of the $\operatorname{Re}(P)$ at zero field exceeds that of the $1 \mathrm{LO}$ peak ( 0.56 for $2 \mathrm{LO}$ against 0.41 for $1 \mathrm{LO}$ ). Second, the polarization degree varies more slowly with increasing exciton splitting. If we assume that the $2 \mathrm{LO}$ peak represents the next step of the cascade relative to that of the $1 \mathrm{LO}$ peak, we should expect the contrary. This fact suggests that the 2 LO peak may arise not only as the successive step to the 1 LO one in a cascade process, but also directly from the photo-created exciton state by simultaneous emission of two phonons. The $2 \mathrm{LO}$ peak in $\mathrm{ZnCrTe}$ also shows anomalies. The value of $\operatorname{Re}(P)$ starts from 0.3 at zero splitting and rises to 0.45 at maximum splitting. The reason of this fact is up to now not clear.

\subsection{CdMnTe quantum well}

In SMSC quantum wells made of CdMnTe and nonmagnetic barriers made of CdMgTe, under a magnetic field of several teslas in Voigt configuration, multiple $\mathrm{Mn}^{2+}$ spin-flip scattering has been observed by Stühler et al. [7]. The Raman spectra taken at $2 \mathrm{~K}$ consisted of series of peaks corresponding to spin resonance with $g=2$. Under excitation of linearly polarized laser beam, no polarization of the scattered light was seen. As the temperature rose, the linear polarization appeared, with alternate sign between two consecutive peaks.

We used an MBE-grown sample consisting of four quantum wells made of $\mathrm{Cd}_{0.976} \mathrm{Mn}_{0.024} \mathrm{Te}$, with well widths of $100,40,20$ and $13 \AA$, separated by $500 \AA$ non-magnetic barriers made of $\mathrm{Cd}_{0.86} \mathrm{Zn}_{0.14} \mathrm{Te}$. The measurements were carried out at $36 \mathrm{~K}$. At this temperature the intensity of the peaks is still strong enough, and the polarization degree is sufficiently high to be measured. In order to study the resonant Faraday effect, we created a longitudinal magnetic field component by deviating the sample plane from the direction of the magnetic field. To calibrate this field component, the exciton Zeeman splitting was determined from reflectivity measurements in the Faraday configuration, and at the same temperature, as a function of the field magnitude.

The results obtained for the $40 \AA$ quantum well are shown in Fig. 2, where we present the real and imaginary parts of $P$ for the three most intensive peaks (numbered 1,2, and 3 ) as a function of the effective exciton Zeeman splitting arising from the deviation of the sample plane. The sign of the polarization degree for all the three peaks is taken to be the same for the sake of clearness of the figure. The main features of the curves are as follows: all the peaks have nearly the same value of polarization degree at each exciton splitting value, and the dependence 
of the polarization degree on exciton splitting is identical for all the peaks. With the increase in the exciton splitting, $\operatorname{Re}(P)$ decreases, changes its sign at exciton splitting value of about $4 \mathrm{~cm}^{-1}$, and continues to increase in absolute value, while $\operatorname{Im}(P)$ starts from zero, increases in absolute values, reaches its maximum at exciton splitting of about $3.7 \mathrm{~cm}^{-1}$ and then decreases.

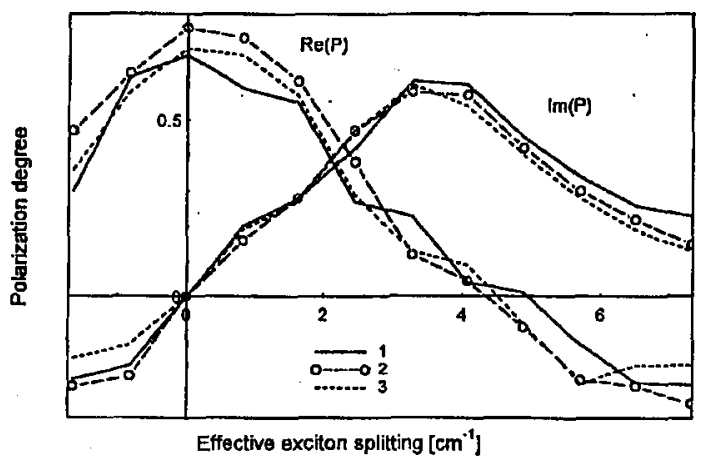

Fig. 2. Polarization degree of three Raman peaks in $40 \AA$ wide CdMnTe quantum well vs. effective exciton splitting.

In order to make an estimation of the characteristic time for the process, we take the value of exciton splitting at which $\operatorname{Re}(P)$ changes its sign and/or $\operatorname{Im}(P)$ has a maximum. This gives the value of this time as $T \approx 1.3 \mathrm{ps}$.

The similarity of the depolarization curves for the three peaks corresponding to different scattering orders clearly shows that the mechanism of their formation cannot be explained by a cascade process.

\section{Acknowledgments}

This work was partially supported by the Committee for Scientific Research by the grant $8 \mathrm{~T} 11 \mathrm{~B} 01411$.

\section{References}

[1] M. Nawrocki, R. Planel, C. Benoît à la Guillaume, Phys. Rev. Lett. 36, 1343 (1976).

[2] J.A. Gaj, Nguyen The Khoi, M. Nawrocki, Ann. Phys. (France), Col. C2, Suppl. au no. 320, C.2-79 (1995).

[3] D. Scalbert, J.A. Gaj, A. Mauger, J. Cernogora, C. Benoît à la Guillaume, A. Mycielski, J. Cryst. Growth 101, 940 (1990).

[4] J.A. Gaj, Nguyen The Khoi, M. Nawrocki, A. Golnik, K. Lebecki, Acta Phys. Pol. $A$ 80, 409 (1991).

[5] Nguyen The Khoi, W. Mac, J.A. Gaj, Acta Phys. Pol. A 82, 634 (1992).

[6] E.I. Ivchenko, G.E. Pikus, B.S. Razbirin, A.I. Starukhin, Zh. Eksp. Teor. Fiz. 72, 2230 (1977) [Sov. Phys. JETP 45, 1772 (1977)].

[7] J. Stühler, G. Schaack, M. Dahl, A. Waag, G. Landwehr, K.V. Kavokin, I.A. Merkulov, Phys. Rev. Lett. 74, 2567 (1995).

[8] W. Mac, Nguyen The Khoi, A. Twardowski, M. Demianiuk, J. Cryst. Growth 159, 993 (1996). 